\title{
Natural flavonoids as superior reagents for separation of clinically important $\mathrm{Zr}$ radionuclides
}

\author{
Kalpita Ghosh ${ }^{\mathrm{a}}$, Nabanita Naskarb ${ }^{\mathrm{b}}$, Dibyasree Choudhury ${ }^{\mathrm{b}}$, Susanta Lahirib,c* \\ ${ }^{a}$ Charuchandra College, 22, Lake Place Road, Lake market, Kalighat, Kolkata-700029 \\ bSaha Institute of Nuclear Physics, 1/AF, Bidhannagar, Kolkata-700064. \\ ${ }^{\mathrm{c}}$ Homi Bhabha National Institute, India \\ *Corresponding Author: susanta.lahiri@saha.ac.in
}

\begin{abstract}
This paper exploits the affinity of bio-flavonoid hesperidin, isolated from orange peel, towards the separation of clinically important ${ }^{88} \mathrm{Zr}$ radionuclide from high energy proton bombarded yttrium target. The extraction of $\mathrm{Zr}$ with hesperidin was studied by solid-liquid extraction (SLX) technique over a wide $\mathrm{pH}$ range. The advantage of hesperidin is that it acts as separating agent as well as free-radical scavenging agent upon injection of the radionuclidehesperidin complex in-vivo. The stability of isolated hesperidin under strong radiation field was also studied. The selectivity of the bio-flavonoid towards ${ }^{88} \mathrm{Zr}$ was maximum at $\mathrm{pH}=4$ where in $>78 \% \mathrm{Zr}$ was extracted with $<1 \%$ contamination from bulk yttrium target.
\end{abstract}

Keywords: Flavonoid; Hesperidin; PET-isotopes; Separation

\section{Introduction}

Nature is enriched with resources of large numbers of molecular entities, which can be obtained from different terrestrial flora. Nature resourced chemistry in the framework of green chemistry can be defined as the development of chemical processes with the help of the chemicals or reagents of natural origin'. The philosophy and mandate green chemistry has crossed the boundary of chemical sciences and widened its scope to many other branches of 
sciences. This paper deals with separation of clinically upcoming and important positron emitting radioisotopes ${ }^{88,89} \mathrm{Zr}$ from bulk $\mathrm{Y}$ target by hesperidin, a flavonoid compound of the flavanone group isolated from the orange peels. The inspiration behind the use of hesperidin as a separation reagent is the free radical scavenging property of metal-flavonoid complex. Free radical generation is always associated with the in vivo use of radionuclides. At molecular level, free radicals destroy biologically active molecules by either removing electrons or removing hydrogen atoms eventually leading to cell death. The anti-oxidant property of bio-flavonoids is displayed by donation of electrons from their hydroxyl groups to the free radicals. Long term investigation with 40 metal complexes of flavonoids demonstrated that the free radical scavenging properties of metal-flavonoid complexes are stronger than the free flavonoids due to enhanced physiological properties of flavonoids upon metal binding ${ }^{2}$. Therefore this experiment demonstrates a high manifestation of green chemistry mandates.

Positron emission tomography (PET) is an established non-invasive, three-dimensional nuclear imaging technique that offers the advantage of tracer quantification with its high sensitivity, accuracy, resolution, short time imaging protocols and lower radiation exposure ${ }^{3}$ The radionuclides, such as ${ }^{68} \mathrm{Ga},{ }^{64} \mathrm{Cu}$ and ${ }^{89} \mathrm{Zr}$, can be rapidly complexed by chelatorconjugated vectors like small peptides or proteins in nearly quantitative yields ${ }^{4}$. The suitable nuclear characteristics of ${ }^{89} \mathrm{Zr}$ (supplementary Table-1) makes it a promising radionuclide in nuclear medicine for immuno-PET, bio-distribution studies, tracking and quantification of monoclonal antibodies (mAbs) ${ }^{5}$.Ever since its first utilization by Link et al in $1986,{ }^{89} \mathrm{Zr}-$ based PET imaging has been investigated for a wide variety of cancer-related targets, 
including human epidermal growth factor receptor 2, prostate-specific membrane antigen, splice variant v6 of CD44, vascular endothelial growth factor, etc 6 . Another radioisotope of $\mathrm{Zr} ;{ }^{88} \mathrm{Zr}\left(\mathrm{T}_{1 / 2}=83.4 \mathrm{~d}\right)$ is used as tracers for $\mathrm{Zr}$ radiochemistry studies and also for studying the radiochemistry and electrophoretic behavior of $\mathrm{Zr}(\mathrm{IV})$ at nanogram amounts ${ }^{7} .{ }^{88,89} \mathrm{Zr}$ may also be suitable therapeutic radionuclide in cellular level, since both of these radionuclides have high intensity auger electrons (supplementary Table1). The more stable ${ }^{88} \mathrm{Zr}$ may be useful for long-term therapeutic dose at gene level by use of auger therapy. The secondary auger electrons are generally produced in large numbers and their low kinetic energy allows cell damage over a very short range i.e. less than the size of a single cell, in the order of nanometers. This highly facilitates targeted therapies. Thus, production and separation of these isotopes are of utmost importance.

The most feasible pathway for production of ${ }^{89} \mathrm{Zr}$ is via proton bombardment on ${ }^{\text {nat }} \mathrm{Y}$ target through $(\mathrm{p}, \mathrm{n})$ reaction ${ }^{5,8-15}$. Separation techniques based on solvent extraction ${ }^{8,10,15-18}$, cation and anion exchange chromatography ${ }^{8}$, and solid based hydroxamate resins ${ }^{5,11}$ are reported. A separation of Zr present in the rubble waste generated at the Fukushima Daiichi nuclear power station was done using trans uranium resin (TRU) and tetravalent actinide resin (TEVA) ${ }^{19}$. Over a decade, our continuous endeavour was to use green chemistry mandates in radiochemical experiments ${ }^{20}$. Earlier, in 'Radio-green Chemistry' experiments we have used many environmental friendly benign bio reagents like Poly (N-vinylpyrrolidone) $)^{21-22}$, polyethylene glycol ${ }^{23-24}$ and many plant biomaterials ${ }^{25-27}$. 
Many reports on the complex formation of zirconium are reported but zirconium cation is a bone seeker and unstable complex formation is likely to increase $\mathrm{Zr}$ in the skeletal system. This is a matter of concern from dosimetry point of view. Thus together with the demand of using a multidentate ligand which may form stable complex with $\mathrm{Zr}$ and the urge to follow the mandates of green chemistry, in this paper, we have attempted separation of no-carrier-added (NCA) ${ }^{88} \mathrm{Zr}$ from proton irradiated natY target using natural hesperidin (Fig.1) isolated from orange peel. The separation method carried out with ${ }^{88} \mathrm{Zr}$ would be equally valid for ${ }^{89} \mathrm{Zr}$ since both possess similar chemical properties. The metal binding sites of hesperidin are marked in Fig. 1 as $\mathrm{A}, \mathrm{B}$ and $\mathrm{C}$; the major binding site is the carbonyl group ' $\mathrm{C}$ '. The bonding with metal occurs by electron transfer from the $d$ orbital of the metal ion to the $\Pi^{*}$ orbital of the flavonoid. The optimal $\mathrm{pH}$ for complex formation is around $\mathrm{pH}$ 6. Complex formation at $\mathrm{pH}$ values less than 3 is difficult because flavanoid exist predominantly in their un-dissociated form. The properties of hesperidin have been listed in Supplementary-Table 2.

\section{Experimental}

2.1 Materials : ${ }^{\text {nat }}$ Y foil $(25 \times 25 \mathrm{~mm}, 99.9 \%)$ was purchased from Alfa Aeser. Petroleum ether emplura, Methanol GR, glacial acetic acid (99-100\%), conc. $\mathrm{H}_{2} \mathrm{SO}_{4}, \mathrm{~N}, \mathrm{~N}-\mathrm{Dimethyl}$ formamide (DMF) GR and suprapur $\mathrm{HCl}(30 \%)$ were purchased from MERCK, India. Acetonitrile (99.9\%) was procured from Spectrochem Pvt.Ltd, Mumbai. Hesperidin standard ( $>97 \%$ HPLC grade) was procured from Sigma Aldrich. Zirconium oxy-chloride was purchased from Loba-Chemie. De-ionized water $(18.2 \mathrm{M} \Omega \mathrm{cm})$ obtained from Thermoscientific Barnstead Nanopure water purification system was used throughout the experiment. 
2.2 Production of ${ }^{88} \mathrm{Zr}$ and ${ }^{88} \mathrm{Y}$ tracers: Details of production of ${ }^{88} \mathrm{Zr}$ and ${ }^{88} \mathrm{Y}$ tracers and the gamma spectrometric method have been provided in supplementary information (Section 1).

2.3 Isolation of hesperidin: Hesperidin was isolated from orange peel, a natural resource using the established method ${ }^{28} .50 \mathrm{~g}$ sun-dried and powdered orange peel was taken in a round-bottomed flask attached to a reflux condenser. About $250 \mathrm{~mL}$ of petroleum ether (BP 40-60 ${ }^{\circ} \mathrm{C}$ ) was added and heated on a water bath for $1 \mathrm{~h}$. The solution was filtered while hot and the residue was dried at room temperature. The dry powder was transferred to the flask with the addition of $250 \mathrm{~mL}$ of methanol. The content was refluxed for $3 \mathrm{~h}$ and then filtered hot. It was further washed with $50 \mathrm{~mL}$ hot methanol. The filtrate was concentrated and the syrupy residue was crystallized from dilute acetic acid, yielding white needles of hesperidin (http://www.chemicalbook.com/ProductMSDSDetailCB3234127_EN.htm). A schematic diagram for isolation of hesperidin has been provided in Fig. 2.

2.4 Radiation stability of hesperidin: The radiation stability of hesperidin was studied as a function of absorbed dose upto $125 \mathrm{kGy}$. The solid sample was irradiated in a ${ }^{60} \mathrm{Co}$ gamma chamber (GAMMA CHAMBER 1200, BRIT, Mumbai) of dose rate $1.9 \mathrm{kGy} / \mathrm{h}$ for $66 \mathrm{~h}$ at UGC-DAE-CSR, Kolkata. To study the possibility of radiolytic degradation of hesperidin under the influence of ${ }^{60} \mathrm{Co}$ radiation field, it was dissolved in DMF and monitored by UV-Vis spectroscopic studies using JASCO V-780 spectrophotometer. 


\subsection{Identification of hesperidin extracted from orange peels:}

(a) Melting point measurement: The melting point of un-irradiated hesperidin and $\gamma$-ray irradiated hesperidin was determined in melting point apparatus.

(b) High Pressure Liquid Chromatography (HPLC): Reversed Phase HPLC technique was performed to verify the purity of the extracted hesperidin. HPLC Surveyor Plus from Thermo Fisher Scientific equipped with vacuum degasser, a quaternary pump programmable for making gradients, injection valve with a $20 \mu \mathrm{L}$ sample loop and UV-Vis detector with dual wavelength was employed for the study. All the modules were computer controlled with interface and HPLC System Manager window-based software Chrom Quest4.2. Wavelength adopted for this study was $280 \mathrm{~nm}$. C-18 syncronis column $(250 \mathrm{~mm} \times 4.6 \mathrm{~mm})$ of particle size $5 \mu \mathrm{m}$ fitted with suitable guard column was used. The mobile phase adopted was acetonitrile (isocratic) with a flow rate of $0.5 \mathrm{~mL} / \mathrm{min}$ and temperature $30^{\circ} \mathrm{C}$. Solutions of standard hesperidin and extracted hesperidin were prepared in DMF. The purity of hesperidin extracted from orange peels was compared with chromatogram of standard hesperidin solution.

2.6 Separation of ${ }^{88} \mathrm{Zr}$ from bulk $\mathrm{Y}$ : The irradiated $\mathrm{Y}$ foil was dissolved in minimum volume of $1 \mathrm{M} \mathrm{HCl}$. Hesperidin, being insoluble in aqueous medium it was possible to carry out solid liquid extraction (SLX) with $\mathrm{HCl} .10 \mathrm{mg}$ of hesperidin was added separately to 1.5 $\mathrm{mL} \mathrm{HCl}$ of varying concentrations from $10^{-4} \mathrm{M}$ to $6 \mathrm{M}$. To each of this set, $0.1 \mathrm{~mL}$ activity was added and was shaken for 10 minutes, centrifuged at $5000 \mathrm{rpm}$ for 5 minutes and allowed to settle. $1 \mathrm{~mL}$ supernatant was taken in eppendorf to assay the radionuclides from each solution counted in HPGe detector for $1000 \mathrm{~s}$ and was compared with the initial activity, prepared in the same way without adding hesperidin. 


\section{Results and Discussions}

The $\gamma$-spectrum taken after 7 days of the EOB showed the presence of ${ }^{89} \mathrm{Zr}\left(\mathrm{T}_{1 / 2}^{1 / 2}=3.3 \mathrm{~d}\right),{ }^{88} \mathrm{Zr}$ $\left(\mathrm{T}_{1 / 2}=83.4 \mathrm{~d}\right)$ and ${ }^{88} \mathrm{Y}\left(\mathrm{T}_{1 / 2}=106.61 \mathrm{~d}\right)$ produced through ${ }^{89} \mathrm{Y}(\mathrm{p}, \mathrm{n}),{ }^{89} \mathrm{Y}(\mathrm{p}, 2 \mathrm{n})$ and ${ }^{89} \mathrm{Y}(\mathrm{p}, \mathrm{pn})$ reactions (Fig. 3). Another gamma spectrum taken 6 months after EOB (Fig. 4) indicated the presence of only ${ }^{88} \mathrm{Zr}$ and ${ }^{88} \mathrm{Y}$.

Radiation stability studies of hesperidin is important since in the real scenario extraction using hesperidin as the solid exchanger would be carried out in highly radioactive solutions containing ${ }^{88,89} \mathrm{Zr}$ and ${ }^{88} \mathrm{Y}$. The UV-Vis spectrum of both irradiated and non-irradiated hesperidin (Fig. 5) revealed that hesperidin is stable even after getting exposed to $125 \mathrm{kGy}$ dose of $\gamma$ - rays obtained from ${ }^{60} \mathrm{Co}$ source and do not dissociate. The UV-Vis spectrum of both the solutions showed the same absorption maxima at $285 \mathrm{~nm}$ and $336 \mathrm{~nm}$. Melting point of both $\gamma$ - ray irradiated and non-irradiated hesperidin was $\sim 252{ }^{\circ} \mathrm{C} \pm 1{ }^{\circ} \mathrm{C}$ which further confirmed that hesperidin is stable when exposed to radiation.

HPLC analysis of the extracted hesperidin in DMF revealed a single peak in the chromatogram with retention time $\left(\mathrm{R}_{\mathrm{T}}\right)$ of $7.21 \mathrm{~min}$ with acetonitrile (isocratic) as mobile phase. The $\mathrm{R}_{\mathrm{T}}$ of extracted hesperidin coincided with the $\mathrm{R}_{\mathrm{T}}$ of standard (7.19 min).The HPLC chromatogram of extracted hesperidin has been shown in Fig. 6. A single peak in chromatogram justifies the purity of the sample.

To verify whether red shift occurs upon binding of $\mathrm{Zr}$ with hesperidin, we carried out similar SLX with stable $\mathrm{Zr}$ in the form of aqueous solution $(0.14 \mathrm{M})$ of zirconium oxy-chloride salt; 
$\mathrm{ZrOCl}_{2} \cdot 2 \mathrm{H}_{2} \mathrm{O}$. Experiment with radioactive tracer revealed the extraction of $\mathrm{Zr}$ by hesperidin, hence the solid hesperidin phase was dissolved in DMF and studied in JASCO V-780 spectrophotometre (Figure 7). A $5 \mathrm{~nm}$ red shift was observed.

The extraction profile of $\mathrm{NCA}{ }^{88} \mathrm{Zr}$ and bulk $\mathrm{Y}$ by hesperidin from different concentration of $\mathrm{HCl}$ is shown in the Fig. 8. At lower acid concentration, i.e., at $10^{-4} \mathrm{M} \mathrm{HCl}$, high separation between NCA $\mathrm{Zr}$ radionuclides and bulk $\mathrm{Y}$ was achieved. About $78 \%{ }^{88} \mathrm{Zr}$ was extracted by hesperidin with $<1 \%$ contamination from bulk Y. After multiple extractions, $>95 \% \mathrm{Zr}$ will be extracted in the hesperidin phase without contamination from bulk Y. The entire experimental procedure has been schematically shown in Fig. 9.

It is evident from the Fig. 8 that the extraction of $\mathrm{Zr}$ varies with the acidity of the medium while the extraction of $\mathrm{Y}$ is negligible throughout the acid range. The extraction behavior may be explained by considering the speciation of $\mathrm{Y}$ and $\mathrm{Zr}$ at different $\mathrm{H}^{+}$concentrations with the help of the software CHEAQS ${ }^{29}$. Zr exists in different species while $\mathrm{Y}$ exists predominantly as free $\mathrm{Y}^{3+}$ throughout the entire acid range. The phenolic $\mathrm{OH}$ groups of hesperidin preferentially interact with $\mathrm{Zr}$ probably through complexation, and therefore $\mathrm{Zr}$ is extracted by hesperidin. At $\mathrm{pH} 4, \mathrm{Zr}$ exists as neutral zirconium hydroxide by $99.91 \%$ which could be adsorbed by hesperidin forming chelate. As $\mathrm{pH}$ decreases, the concentration of neutral $\mathrm{Zr}(\mathrm{OH})_{4}$ decreases while that of cationic species such as $\mathrm{Zr}^{4+}, \mathrm{Zr}(\mathrm{OH})_{3}{ }^{+}$, etc., increases. Finally at $3 \mathrm{M} \mathrm{HCl}, \mathrm{Zr}$ exists as $\mathrm{Zr}(\mathrm{OH})^{3+}$ in $70 \%$ and $\mathrm{Zr}(\mathrm{OH})_{2}{ }^{2+}$ in $30 \%$ with $0 \%$ contribution from neutral $\mathrm{Zr}(\mathrm{OH})_{4}$. As the existence of $\mathrm{Zr}$ in neutral form decreases, the extraction of $\mathrm{Zr}$ by hesperidin also decreases. 
The developed method for separation of NCA $\mathrm{Zr}$ and bulk yttrium target has multiple advantages (i) the separation has been carried out by hesperidin and the Zr-hesperidin complex itself can directly be used for in vivo use. ${ }^{88} \mathrm{Zr}$-hesperidin complex would act as a free radical scavenger (ii) the technique is based on a naturally obtained chemical compound which is interesting from green chemistry point of view (iii) the separation is simple, rapid as well as economical (iv) this method utilizes the fact the metal-hesperidin complex acts as a better free radical scavenger than free hesperidin and thus eliminates the need of recovery or back extraction of the activity. Radiation stability study of hesperidin followed by melting point measurements confirmed the non-degradation of hesperidin and its existence during the entire extraction process. This experiment may be seen as one which further strengthens that fact that bio-reagents are comparable or even superior to synthetic chemicals ${ }^{26}$

\section{Conflicts of interest}

There are no conflicts to declare

\section{Acknowledgement}

Authors gratefully acknowledge the contribution of Professor Abhijit Saha, Director, UGCDAE-CSR, Kolkata for extending his co-operation, sincere help and all support during our experiment at UGC_DAE_CSR while using the gamma chamber.

\section{References}

1. S.Lahiri, D. Choudhury and K.Sen, J. Radioanal Nucl Chem., 2018, 318, 1543-1558.

2. V. Kuntić, I. Filipović and Z. Vujić, Molecules, 2011, 16, 1378-1388. 
3. N. Gholipour, M. Akhlaghi, A. Kheirabadi, D. Beiki, P. Geramifar, H. Yousefnia and M. Mazidi, J. Radioanal Nucl Chem., 2017; 311, 1811-1817.

4. A. Schmidt, M. Schottelius, M. Herz and H. Wester, J. Radioanal. Nucl. Chem., 2017, 311; 1551-1557.

5. M. Walther, P. Gebhardt, P.G. Gehling, L. Wurbach, I. Irmler, S. Preusche, M. Khalid, T. Opfermann, T. Kamradt, J. Steinbach and H.P. Saluz, Appl. Radiat. Isotopes, 2011, 69, $852-857$.

6. Y. Zhang, H. Homg and W. Cai, Curr Radiopharm, 2011, 4, 131-139.

7. P. Ivanov, S. Jerome, G. Bozhikov, O. Mastov, G. Starodub and S. Dmitriev; Appl. Radiat. Isotopes, 2014, 90, 261-264.

8. S.A. Kandil, B. Scholten, Z.A. Saleh, A.M. Youssef, S.M. Qaim and H.H. Coenen, J. Radioanal. Nucl. Chem. 2007, 274, 45-52.

9. T. Kakavand, M. Taghilo, Proceedings of the DAE Symp. Nucl. Phys., (Dec 2-6) 2013, 58, 608-609.

10. B. Dutta, M. Maiti and S. Lahiri, J Radioanal Nucl Chem., 2009, 281, 663-667.

11. W. Meijs, J.D.M. Herscheid, H.J. Haisma and H.M. Pinedo., Appl. Radiat. Isotopes, 1994, 45, 1143-1147.

12. I. Verel, G.W. Visser, R. Boellaard, M. Walsum, G.B. Snow, G.A. Dongen, J. Nucl. Med., 2003, 44, 1271-1281.

13. M. Sadeghi, M. Enferadi and M. Bakhtiari, Annals of Nuclear Energy., 2012, 41, 97103.

14. J.P. Holland, Y. Sheh and J.S. Lewis, Nucl. Med. Biol., 2009, 36, 729-739.

15. O. Dejesus and R. Nickles, Appl. Radiat. Isotopes, 1990, 41, 789-790. 
16. J. M. Link, K.A. Krohn, J.F. Eary, R. Kishore, T.K. Lewellen, M.W. Johnson, C.C. Badger, K.Y. Richter and W.B. Nelp, J. Labelled. Comp. Radiopharm., 1986, 23, 1297.

17. A.G. Espartero, J.A. Suarez, M. Rodriguez and G. Pina, Appl. Radiat. Isotopes, 2002, 56, 41-46.

18. S. Lahiri, B. Mukhopdhyay and N.R. Das, J. Radioanal. Nucl. Chem., 1997, 48, 883886.

19. A. Shimada, Y. Kameo, J. Radioanal. Nucl. Chem., 2017, 311, 1613-1618.

20. S. Lahiri, J. Radioanal. Nucl. Chem.; 2016, 307, 1571-1586.

21. S. Lahiri, S. Sarkar, Appl. Radiat. Isotopes, 2007a, 65, 309-312.

22. S.Lahiri, S. Sarkar, Appl. Radiat. Isotopes, 2007b, 65, 387-391.

23. K. Roy, S. Lahiri, Radiochim. Acta, 2008, 96, 49-53.

24. A. Datta, M. Maiti and S. Lahiri, J. Radioanal Nucl Chem., 2014, 302, 931-937.

25. K. Ghosh and S. Lahiri, J. Radioanal. Nucl. Chem., 2007, 274, 233-236.

26. D. Nayak, K. Hazra, S. Laskar and S. Lahiri, J. Radioanal. Nucl. Chem., 2008, 275, 423-426.

27. K. Roy, K. Ghosh, A. Banerjee, D. Mukherjee, S. Lahiri, Biochem. Eng. Journ., 2009, 45, 82-85.

28. R. Ikan, Natural Products (A Laboratory Guide) $2^{\text {nd }}$ Edition, Academic Press Inc, USA, 1991, 10.

29. W. Verweij, W, http://home.tiscali.nl/cheaqs/ (2005) 


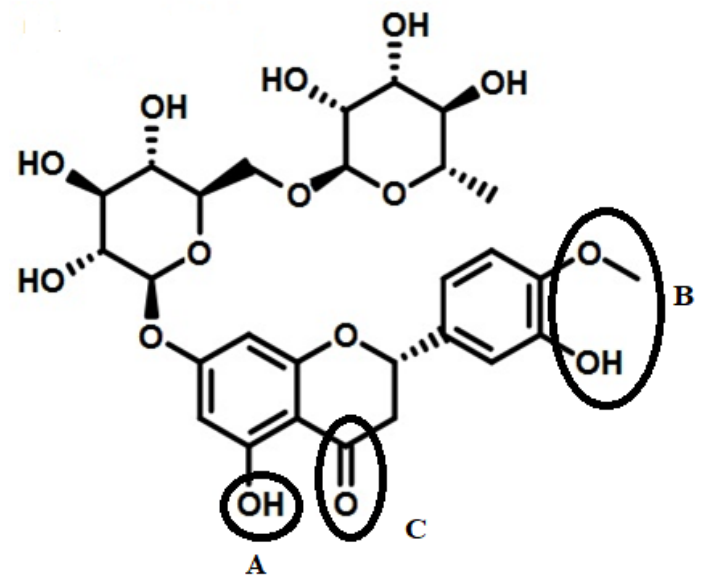

Fig.1 Structure of hesperidin (www.chemicalbook.com)

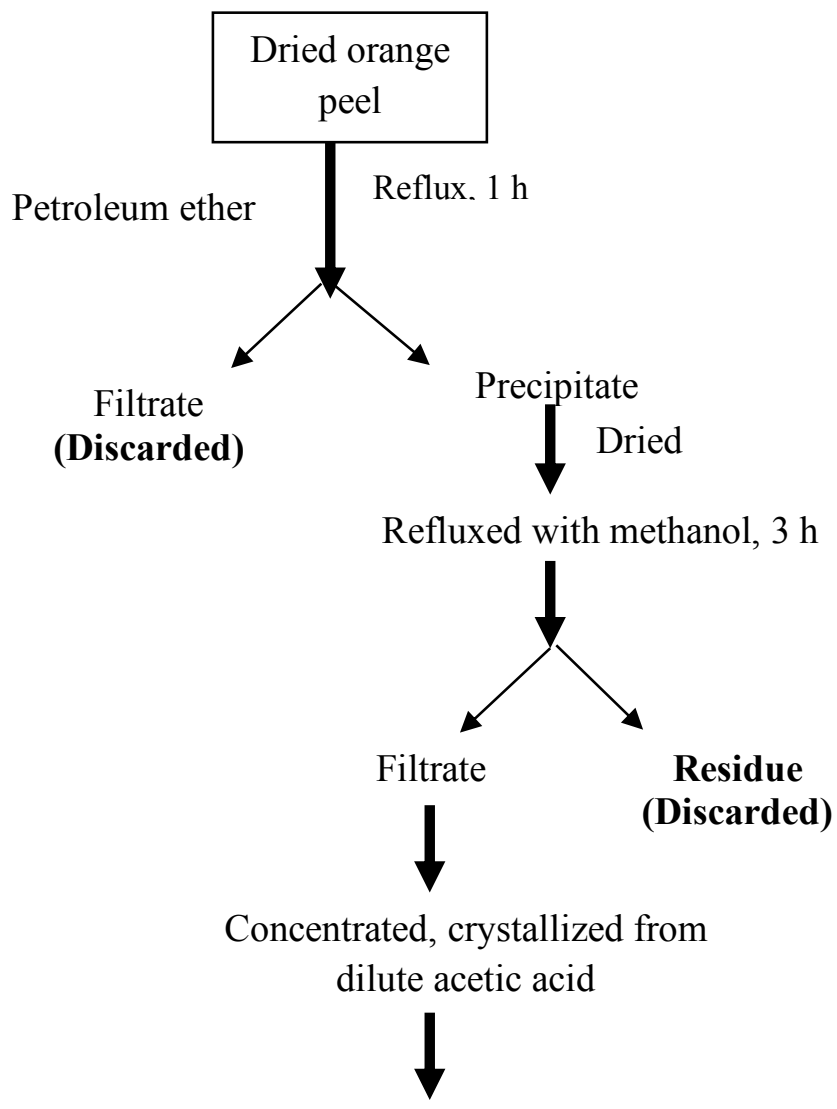

Hesperidin

Fig. 2 A schematic diagram for isolation of hesperidin 


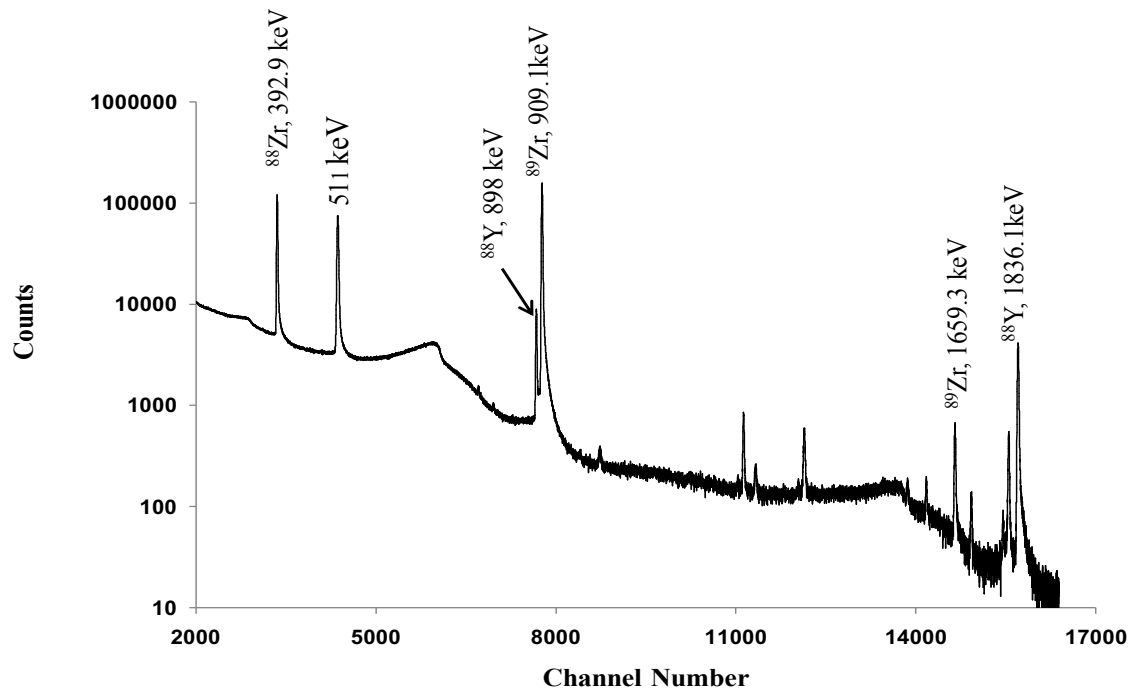

Fig. 3 Gamma spectrum taken 7 d after EOB

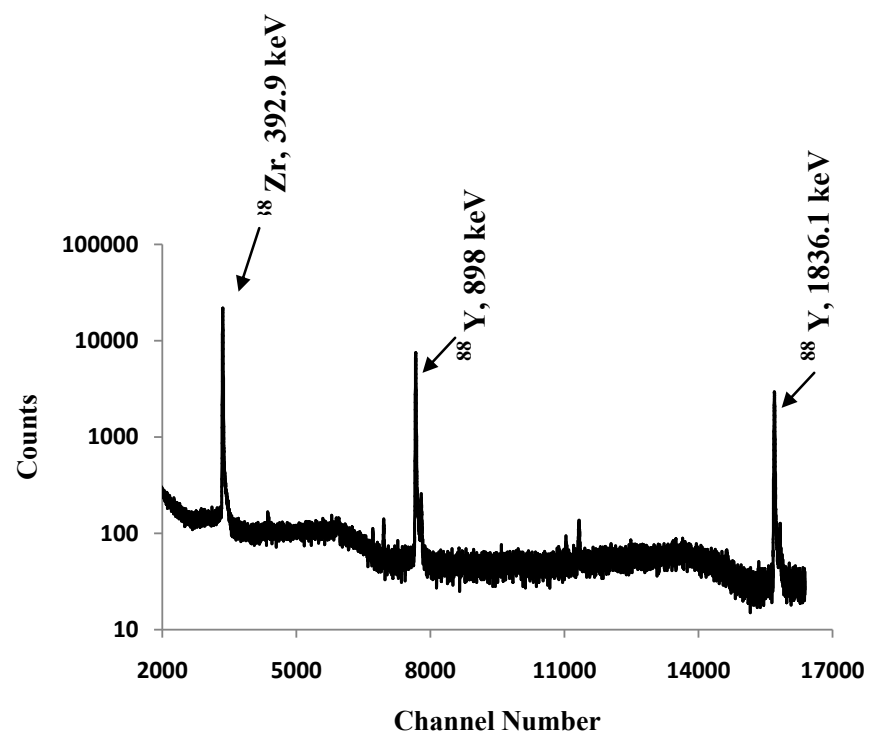

Fig. 4 Gamma spectrum taken 6 months after EOB 


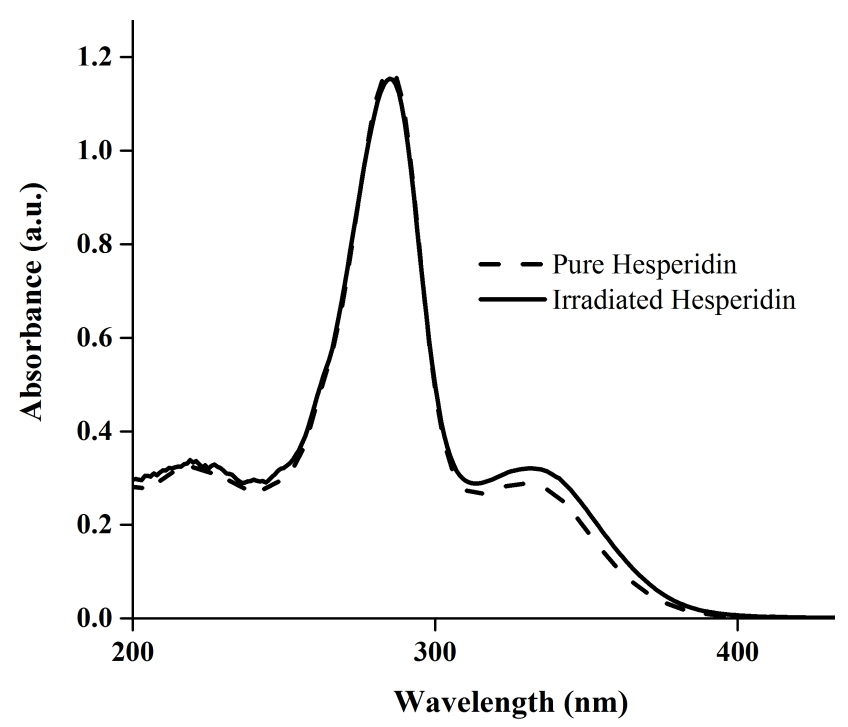

Fig. 5 Absorption spectrum of pure hesperidin and ${ }^{60} \mathrm{Co}$ irradiated hesperidin in DMF

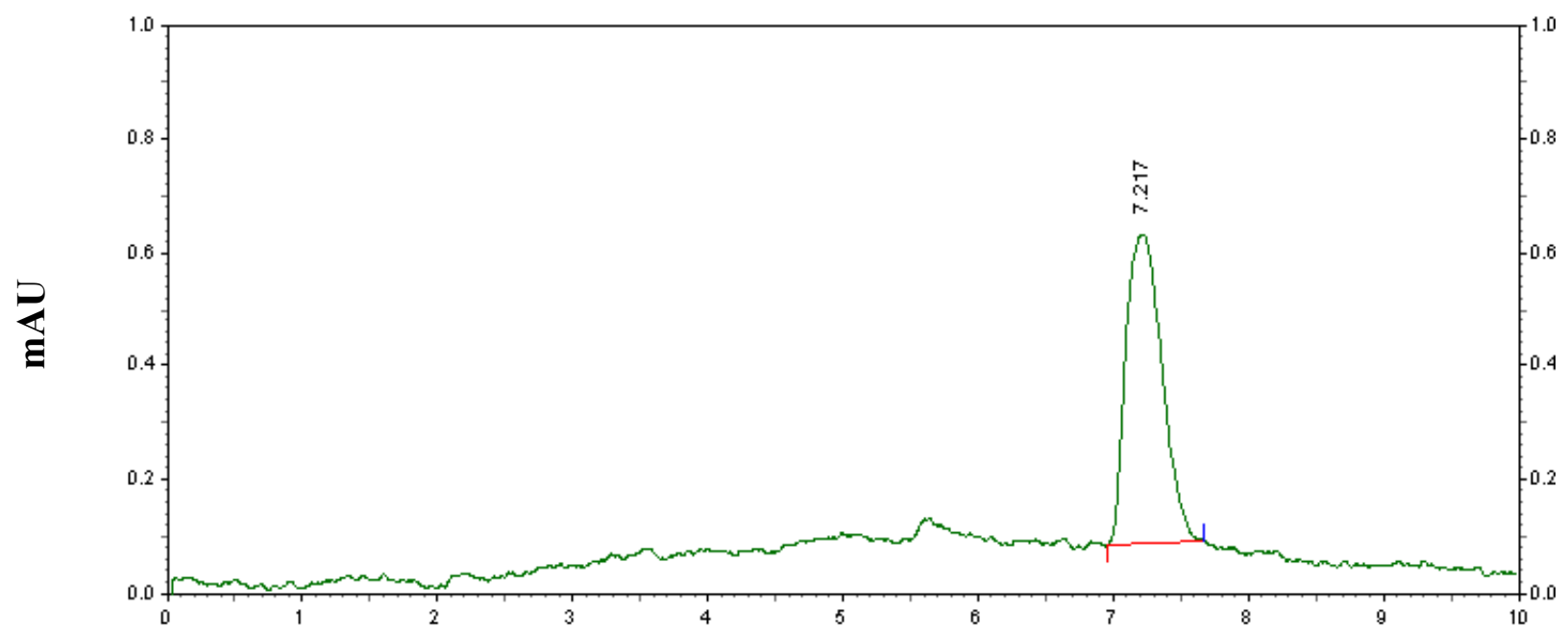

Minutes

Fig. 6 HPLC spectrum of hesperidin extracted from orange peel 


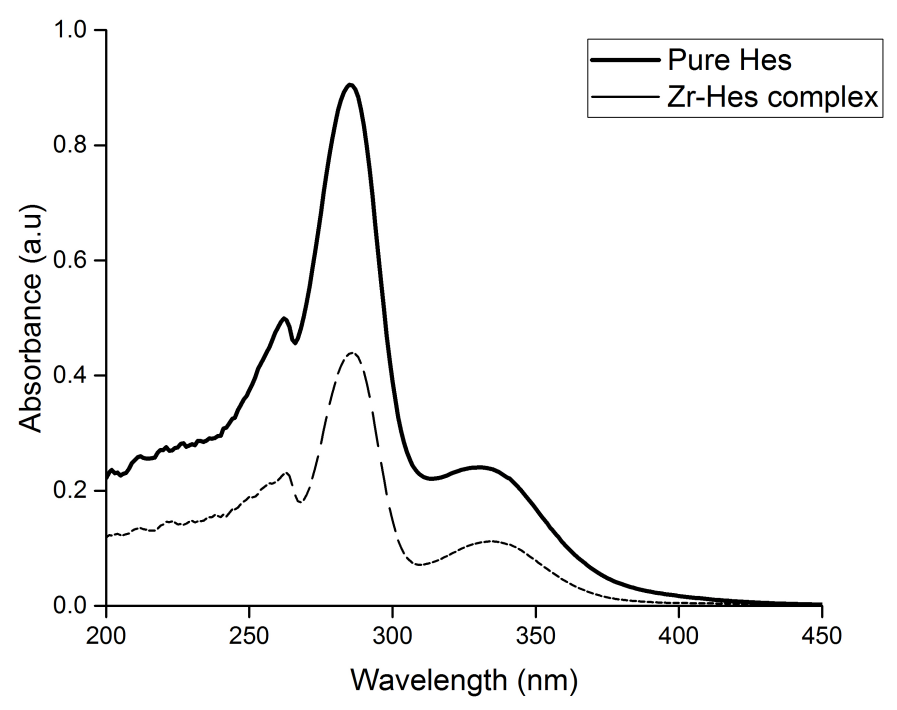

Fig. 7 Red shift validation of hesperidin upon metal binding

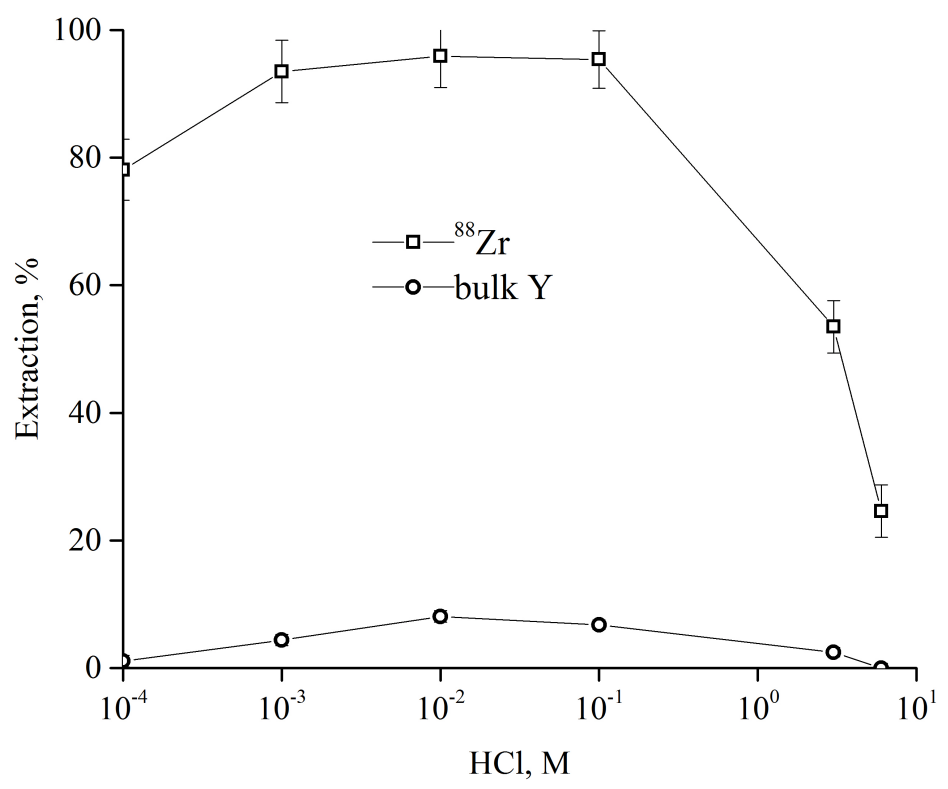

Fig. 8 Extraction profile of ${ }^{88} \mathrm{Zr}$ and bulk $\mathrm{Y}$ by hesperidin at different $\mathrm{pH}$ 


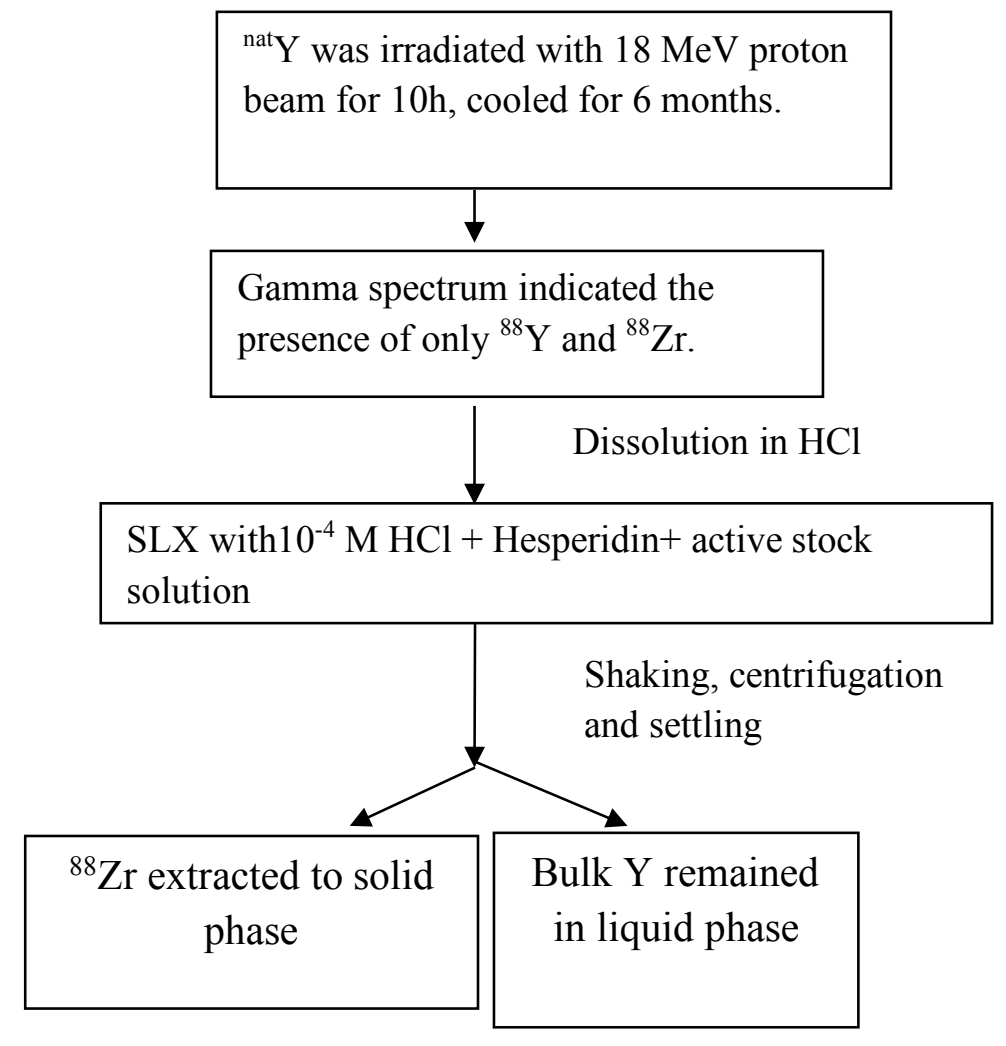

Fig. 9 Schematic diagram of the experimental procedure 